\title{
Graphael: A System for Generalized Force-Directed Layouts ${ }^{\star}$
}

\author{
David Forrester, Stephen G. Kobourov, Armand Navabi, \\ Kevin Wampler, and Gary V. Yee \\ Department of Computer Science, \\ University of Arizona \\ \{forrestd, kobourov, navabia, wamplerk, gyee\}@cs.arizona.edu
}

\begin{abstract}
The graphael system implements several traditional forcedirected layout methods, as well as several novel layout methods for non-Euclidean geometries, including hyperbolic and spherical. The system can handle large graphs, using multi-scale variations of the forcedirected methods. Moreover, graphael can layout and visualize graphs that evolve though time, using static views, animation, and morphing. The implementation includes a powerful interface that allows the user to put together existing algorithms and visualization techniques, and to easily add new ones. The system is written in Java and is available as a downloadable program or as an applet at http://graphael.cs.arizona.edu.
\end{abstract}

\section{Introduction}

As researchers in the graph drawing community develop new algorithms and visualization techniques it is natural for the creation of new graph drawing tools to follow. It is often the case, however, that the implementation of an algorithm is accompanied by time consuming tasks that have little to do with the algorithm itself. Researchers who would like to test a new layout algorithm should only have to concern themselves with the details of the algorithm itself rather than with graphics packages, file parsers, or user interface design.

In this paper we present graphael: yet another graph drawing system designed to provide the necessary structure and flexibility for force-directed graph drawing research. Our system is built with the following design considerations: (1) Plug-and-Play: it should be easy to integrate new algorithms and visualization methods; (2) User Friendliness: the user interface should be easy to use, but also powerful and versatile; (3) Portability: the system should run on any computational platform.

The graphael system attempts to meet these goals by providing a set of core algorithms and visualization routines, as well as an interface that allows the user to combine different algorithms and visualization methods, and to easily add new ones. In addition, the system contains several novel algorithms and visualization techniques, such as force-directed methods in non-Euclidean geometries, and techniques for dealing with graphs that evolve through time.

* This work is supported in part by the NSF under grant ACR-0222920 and ITCDI under grant 003297. 


\subsection{Related Work}

A number of automated graph drawing systems have been developed over the last few years; see [9] for a survey. The GraphServer [3] is an online service that allows users to draw graphs and translate graph descriptions between multiple formats. Tulip is a framework built to facilitate large graph drawing research [1]. WilmaScope [4] is a Java application designed specifically for 3D visualization. yFiles [14] is a commercial library of Java classes developed to provide building blocks for graph drawing applications. Pajek [2] is a Windows program designed to handle large graphs for social networks analysis. TGRIP [6] is an extension on the GRIP system [8] and efficiently draws large temporal graphs using intelligent placement. The GraphAEL [5] system extracts three types of evolving graphs from a custom-built graph drawing literature database and creates $2 \mathrm{D}$ and $3 \mathrm{D}$ animations of the evolutions.

\subsection{Our Contributions}

In addition to sharing all the letters with GraphAEL [5], the system described in this paper was inspired by it. We wanted to provide a graph visualization framework that can easily be coupled with the bibliographic database to provide visualizations of the co-citation, collaboration, and topic graphs, produced from the database. This led to the development of the current system, which is equipped with a core package of force-directed algorithms and visualization tools. In addition to putting together well-known algorithms and visualization methods, graphael contains several novel features. Among these features are support for temporal graphs, interactive graph visualization, multi-scale layout algorithms for large graphs, and embedding graphs in non-Euclidean spaces, such as hyperbolic space and spherical space. The current system also includes an interactive Control Flow (CF) Graph, used to put together different combinations of layout algorithms, projections and visualizations, while offering a visual representation of the process.

\section{System Overview}

\subsection{Force-Directed Layouts}

Force-directed layout algorithms are a powerful and practical graph drawing heuristic. They rely on an objective function that maps a particular graph layout to an energy value. Typically such algorithms start with a random drawing of the graph and utilize standard optimization methods to minimize the energy function. The algorithms define functions in which low energies are associated with layouts where adjacent vertices are near some preferred distance from each other, and non-adjacent vertices are well-spaced. The main difference between force-directed algorithms is the choice of energy function and the methods for its minimization.

We have implemented two traditional force-directed algorithms in graphael. The first one is the Fruchterman-Reingold [7] algorithm. It defines an attractive 
force function for adjacent vertices and a repulsive force function for non-adjacent vertices. For a vertex $v, F_{F R}(v)=F_{a, F R}+F_{r, F R}$, where the attractive force is defined as $F_{a, F R}=\sum_{u \in A d j(v)} \frac{\operatorname{dist}_{R^{n}(u, v)^{2}}}{\text { edgeLength }^{2}}(\operatorname{pos}[u]-\operatorname{pos}[v])$ and the repulsive force is defined as $F_{r, F R}=\sum_{u \in A d j(v)} s \cdot \frac{\text { edgeLength }^{2}}{\operatorname{dist}_{R^{n}(u, v)^{2}}} \cdot(\operatorname{pos}[u]-\operatorname{pos}[v])$.

The second force-directed method is the Kamada-Kawai [10] layout algorithm. In this method each pair of vertices connected by a path has forces proportional to the length of the path. The displacement of a vertex $v$ of $G$ is calculated by: $F_{K K}(v)=\sum_{u \in N_{i}(v)}\left(\frac{\operatorname{dist}_{R^{n}(u, v)^{2}}}{\operatorname{dist}_{G}(u, v) \cdot \operatorname{edgeLength}^{2}}-1\right)(\operatorname{pos}[u]-\operatorname{pos}[v])$.

In the above equations, $\operatorname{dist}_{R^{n}}(u, v)$ is the Euclidean distance between $\operatorname{pos}[u]$ and $\operatorname{pos}[v], \operatorname{dist}_{G}(u, v)$ is the graph distance between $u$ and $v$ along a shortest path, edgeLength is the unit edge length, $\operatorname{Adj}(v)$ is the set of vertices adjacent to $v$, and $s$ is a small scaling factor.

\subsection{Multi-scale Graph Drawing}

The effectiveness of force-directed methods rapidly decreases as the input graphs get larger. This is mainly due to the increased difficulty of getting out of local minima and to the runtime complexity, typically quadratic, or cubic in the size of the graph. Multi-scale graph drawing methods address both of these problems by filtering the graph into different levels, called filtration levels, each containing a subset of the initial graph. The levels are laid out from least to most complex. The multi-scale methods rely on good filtrations, good initial placement of the vertices, and on local refinement on each level.

Filtrations: The effectiveness of the multi-scale method depends on each successive filtration level containing a constant fraction of the vertices from the previous level. Thus, good filtrations have $\Theta(\lg n)$ depth and can be quickly computed. In graphael we currently provide three filtration methods: Maximal Independent Set Filtration, Random Graph Filtration, and Cores Filtration:

1. Maximal Independent Set Filtration: A filtration $V=V_{0} \supset V_{1} \supset \ldots \supset V_{k} \supset$ $\emptyset$ of the vertex set $V$ of $G$ is called a maximal independent set filtration if $V_{1}$ is a maximal independent set of $G$, and each $V_{i}$ is a maximal subset of $V_{i-1}$ so that the graph distance between any pair of its elements is at least $2^{i-1}+1$. Maximal Independent Set filtrations have depth $O(\lg n)$ and can be computed in near-linear time [8].

2. Random Graph Filtration: Random filtrations are created by repeatedly removing half of the vertices, chosen at random, starting with the original vertex set $V$ of $G$. The depth of this filtration is also $O(\lg n)$ and the computation time required is linear in the size of $V$. Although simple, this method produces reasonable layouts for large graphs.

3. Cores Graph Filtration: Graph cores are described in [13]. Given a graph $G=(V, E)$, a subgraph $H_{k}=(W ; E \mid W)$ induced by the set $W$ is a $k$-core, or a core of order $k$ if $\forall v \in W: \operatorname{deg}_{H}(v) \geq k$, and $H_{k}$ is the maximum 
subgraph with this property. The core of maximum order is also called the main core. Graph cores can be computed in linear time [2]. If the number of cores is a small constant compared to the size of the graph, we augment the filtration induced by the cores to depth $O(\lg n)$ using the peeling process inherent in the core computation.

Initial Placement and Refinement: The main idea of good initial placement is to add vertices to the current drawing one at a time at a carefully computed position, rather than a random one [8]. For simplicity we describe the process in 2D, but in practice this is done in arbitrary Euclidean, and even some nonEuclidean, spaces. Assume that the highest filtration level has exactly 3 vertices. These vertices are placed at the endpoints of a triangle with sides proportional to the graph distances between the points in the original graph. Vertices in subsequent filtration levels are placed based on their graph distances from already placed vertices from previous filtration levels. The intuition is that if we place the vertices close to their optimal positions initially, the refinement phase will only need a few iterations of a local force-directed calculations to reach a minimal energy state. In graphael's implementation, we use the "3-closest-vertices" strategy. Using this method we place the vertex $t$ at the barycenter of $u, v$, and $w$, the three vertices closest to $t$ from the previous filtration level. Once all the vertices at the current filtration level have been placed, we apply a local forcedirected refinement. The refinement stage is local as for a given vertex $v$ in the current filtration, only a small neighborhood of vertices $N_{i}(v)$ is considered in the force computation.

\subsection{Graphs That Evolve Through Time}

We have also implemented algorithms for visualization of graphs that evolve through time based on techniques described in $[5,6]$. The algorithms are modifications of the standard force-directed algorithms that allow us to deal with vertex-weighted and edge-weighted graphs. Graphs that evolve through time are converted to vertex-weighted and edge-weighted graphs, by treating each instance of the graphs as a timeslice, and connecting neighboring timeslices. The edges connecting different timeslices are called inter-timeslice edges. By changing the weights of these edges, we are able to balance the individual graph readability with the overall mental map preservation between consecutive graphs. Making the inter-timeslice edges heavy, results in fixing the vertex positions in each graph instance. Alternatively, making the inter-timeslice edges light, results in nearly independent layouts of each graph instance.

Weighted Graphs: We modify the force-directed equations for calculating the force vectors to include edge weights and vertex weights so as to place heavy vertices well away from each other and to place vertices connected by heavy edges closer to each other. The unit edge length is modified to $\sqrt{w_{u} \cdot w_{v}} / w_{e}$ for an edge of weight $w_{e}$, connecting vertices $u, v$ of weight $w_{u}, w_{v}$, respectively.

The Kamada-Kawai method relies on the notion of graph distance between pairs of vertices. It is easy to generalize this notion to weighted graphs, but 
because of the computational and space overhead associated with calculating the shortest path between all pairs of vertices in the graph, we use an approximation. Let $p_{1}, p_{2}, \ldots, p_{n}$ be the sequence of vertices in the shortest unweighted path in $G$ connecting two vertices, $u$ and $v$. The modified Kamada-Kawai force vector is given by $F_{K K}(v)=$

$$
\sum_{u \in N_{i}(v)}\left(\frac{2 \cdot \operatorname{dist}_{R^{n}}(u, v)^{2}}{\operatorname{optDist}_{G}(u, v)^{2} \cdot \operatorname{edgeLength}^{2}+\operatorname{dist}_{R^{n}}(u, v)^{2}}-1\right)(\operatorname{pos}[u]-\operatorname{pos}[v]),
$$

where optDist ${ }_{G}(u, v)=\sum_{i=2}^{n} \frac{\sqrt{w_{p_{i}} \cdot w_{p_{i}-1}}}{w_{e_{p_{i} p_{i-1}}}}$. Similarly, we modify the FruchtermanReingold forces as follows: $F_{a, F R}=\sum_{u \in A d j(v)} \frac{w_{e} \cdot \operatorname{dist}_{R^{n}}(u, v)^{2}}{\operatorname{edgeLength}^{2}}(\operatorname{pos}[u]-\operatorname{pos}[v])$ and $F_{r, F R}=\sum_{u \in A d j(v)} s \cdot\left(\frac{\operatorname{edgeLength}^{2} \cdot \sqrt{w_{u} \cdot w_{v}}}{\operatorname{dist}_{R^{n}}(u, v)^{2}}\right)(\operatorname{pos}[u]-\operatorname{pos}[v])$.

Timeslice Attribute: To visualize a series of graphs embodying the evolution of a set of relationships over time, we associate a timeslice attribute with each vertex. The timeslice of a vertex is just a label identifying which graph instance the vertex belongs to. We use the timeslice attribute to partition the vertices of a graph into groups by time. Additional modifications to the force-directed algorithms are needed to accommodate timeslice information.

For the Kamada-Kawai layout method, the function $\operatorname{optDist}_{G}(u, v)$ is $\operatorname{modi}^{-}$ fied so that for two vertices $u, v$ with timeslice indices of $t_{u}$ and $t_{v}$, respectively, is given by: $\operatorname{optDist}_{G}(u, v)=\sum_{i=2}^{n} \delta_{t_{u} t_{v}} \cdot \frac{\sqrt{w_{p_{i}} \cdot w_{p_{i-1}}}}{w_{e}}$, where $p_{1}, p_{2}, \ldots, p_{n}$ is the shortest unweighted path in $G$ connecting two vertices, $u$ and $v$ and $\delta_{t_{u} t_{v}}$ is 1 if $t_{u}=t_{v}$ and 0 otherwise.

The modifications needed for the Fruchterman-Reingold calculations are similar. Repulsive forces are simply eliminated between vertices in different timeslices, $F_{r, w, t, F R}=\delta \cdot F_{r, w, F R}$ while the attractive forces remain unchanged, $F_{a, w, t, F R}=F_{a, w, F R}$.

\subsection{Visualizing Evolving Graphs}

The timeslice information alone is not enough to nicely layout evolving graphs; we must also arrange edges between timeslices so that the layouts can be used for animation. The most straightforward method to animate is simply to use a series of "snapshots" of a graph taken at some interval over a period of time. When visualizing an evolving graph, we would ideally like the graphs of each timeslice to have high readability (i.e. have a pleasing layout) and for consecutive timeslices to be similar, that is, the mental map should be preserved. To meet these constraints the timeslices are combined into a single graph by connecting vertices with the same labels from adjacent timeslices.

Because of the modified optimal distance function, corresponding vertices in different timeslices have no repulsive force on each other, but they still have 


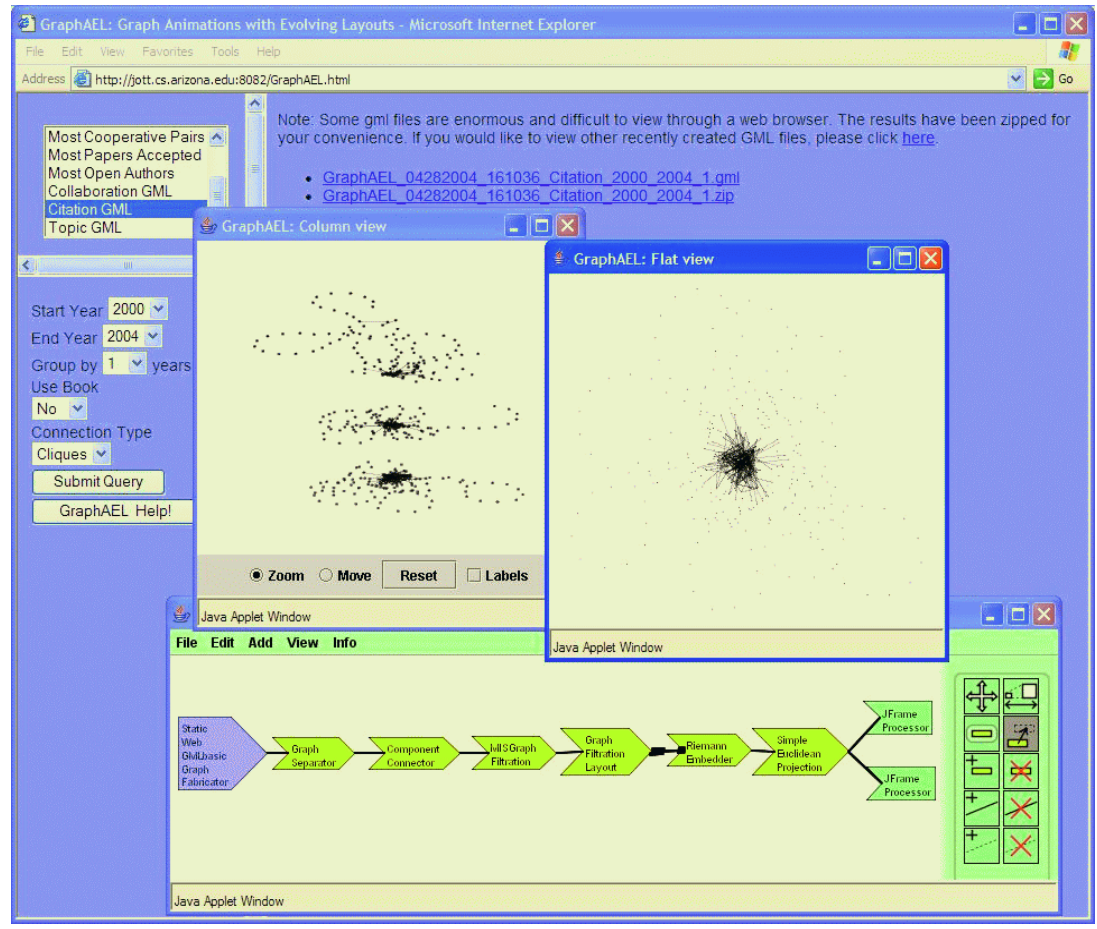

Fig. 1. graphael in cooperation with GraphAEL. The graphs shown are the column view and flat $2 \mathrm{D}$ view of a citation graph from 2000 to 2003 by 1 year increments.

attractive forces due to the inter-timeslice edges. In graphael the balance between readability and mental map preservation can be controlled by changing the weights of the inter-timeslice edges.

Once the layout of the evolving graph has been computed, graphael offers different methods for visualizing the graphs. Each timeslice can be drawn in a restricted $2 \mathrm{D}$ view, or the graphs can be drawn in $3 \mathrm{D}$ with individual graphs arranged on top of each other (column-view). The column view lays out each timeslice on a separate plane, allowing the user to view the changes in the graph over time; see Fig. 1. The inter-timeslice edges can be hidden or displayed. Smoothly stepping through the evolving graphs using linear interpolation of vertex positions, colors, and weights yields visually pleasing animations.

\subsection{Graph Drawing in Non-Euclidean Spaces}

A novel feature in graphael is the option to layout graphs in non-Euclidean spaces, in particular, in hyperbolic space and in spherical space [11]. Existing force-directed algorithms are restricted to calculating a graph layout in Euclidean geometry. Euclidean space has a very convenient structure for force-directed methods. It is easy to define distances and angles, and the relationship between the vector representing the net force on an object and the appropriate motion of 
that object are quite straightforward. Certain non-Euclidean geometries, specifically hyperbolic geometry, have properties which are particularly well suited to the layout and visualization of large classes of graphs [12].

With this in mind we have implemented a generalization of force-directed methods to non-Euclidean geometries that relies on mappings between nonEuclidean geometries and corresponding tangent spaces. While a non-Euclidean geometry does not afford all of the conveniences of Euclidean geometry, there is a straightforward way to define distances and angles, provided we restrict ourselves to geometries which are smooth. Such geometries are known as Riemannian geometries, and while they have less convenient structure than Euclidean geometry, they retain many of the characteristics which are useful for force-directed graph layouts. A Riemannian manifold $M$ has the property that for every point $x \in M$, the tangent space $T_{x} M$ is an inner product space. This means that for every point on the manifold, we can define local notions of length and angle.

Using a local notion of length we can define the length of a continuous curve $\gamma:[a, b] \rightarrow M$ by length $(\gamma)=\int_{a}^{b}\left\|\gamma^{\prime}\right\| d t$. This leads to a natural generalization of the concept of a straight line to that of a geodesic, where the geodesic between two points, $u, v \in M$ is defined as a continuously differentiable curve of minimal length between them. In Euclidean geometry the geodesics are straight lines, and in spherical geometry they are arcs of great circles. Hence, the distance between two points, $d(x, y)$ is defined as the length of the geodesic between them.

Hyperbolic Geometry: Hyperbolic geometry is particularly well suited to graph layout because it has "more space" than Euclidean geometry - in the same sense that spherical geometry has "less space". Unlike in Euclidean geometry, where the relationship between the radius and circumference of a circle in two-dimensional geometry is linear with a factor of $2 \pi$, and constant in a spherical geometry, in hyperbolic geometry the circumference of a circle increases exponentially with its radius. The applicability of this geometric property to graph layout is well-illustrated with the example of a tree. In hyperbolic space, it is possible to layout a tree structure with a uniform distribution of the vertices and with uniform edge lengths despite the fact that the number of vertices at a certain depth in the tree increases exponentially with the depth.

In order to visualize a layout in hyperbolic geometry it is necessary to map the layout into the (2D) Euclidean geometry of a computer monitor. The method used in graphael is the Poincare projection, that maps hyperbolic space onto the open unit disk. The projection compresses the space near the boundary of the unit disk, giving the impression of a fish-eye view. This naturally provides a useful focus + context technique for visualizing the layouts of graphs. This model preserves angles, but distorts lines. A line in hyperbolic space is mapped to a circular arc which intersects the unit circle at right angles. The Poincare disk progressively distorts the graph view as we move away from the center of projection. In Fig. 2(a) we show a drawing of a graph obtained in hyperbolic geometry and displayed in 2D Euclidean space.

Spherical Geometry: Using the same ideas, we can generalize force-directed methods to spherical space. Spherical geometry, like hyperbolic geometry, has 

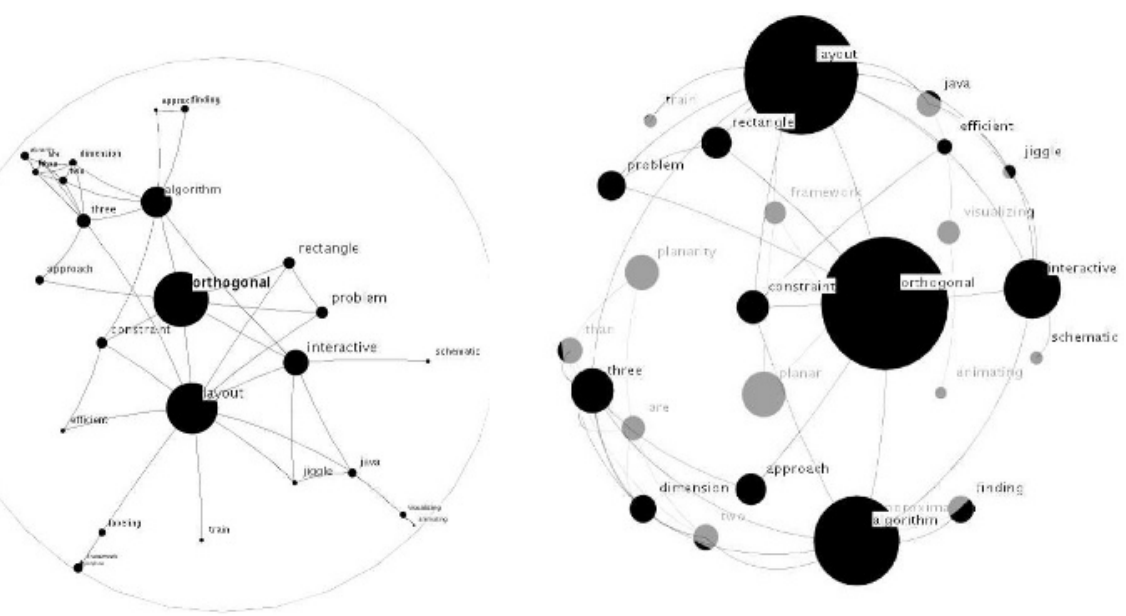

Fig. 2. Layouts of a title-word graph, obtained in Hyperbolic space and in Spherical space. The graph has 27 vertices and 50 edges and the vertices correspond to title-words from papers in the 1999 Graph Drawing conference. The size of a vertex is determined by its frequency and edges are placed between two vertices if they co-occur in at least one paper.

a constant curvature and the equations for mapping to and from the tangent space can be calculated analytically. Each point on a sphere is given a longitude and latitude. The sphere can then be embedded in 3D Euclidean space by a simple parameterization. In Fig. 2(b) we show a drawing of a graph obtained in spherical geometry and displayed in three-dimensional Euclidean space.

Multi-scale Graph Drawing in Non-Euclidean Space: Since we are able to utilize tangent space mapping to use existing force-directed methods for graph layouts, we can also generalize the multi-scale method for drawing large graphs to non-Euclidean spaces. Of the tree stages in the multi-scale method (filtration, initial placement, and refinement) the only stage that requires further consideration is the initial placement stage.

In the initial placement stage we place each vertex one at a time in the barycenter of its neighbors. In Euclidean space we simply take the average of each dimension and place the vertex at that point. For non-Euclidean points we use the mapping to and from a tangent space. Specifically, we map the nonEuclidean points that correspond to the location of the neighbors to a tangent space. From there we are able to calculate the barycenter. We map that back into the non-Euclidean manifold and place the vertex at that location.

\subsection{Graph Editor}

There are several ways to experiment with the graphael system. Loading one of the sample graph files, loading a new file, or creating a new graph. When a user wants to create a graph manually, they have the option of using a basic 


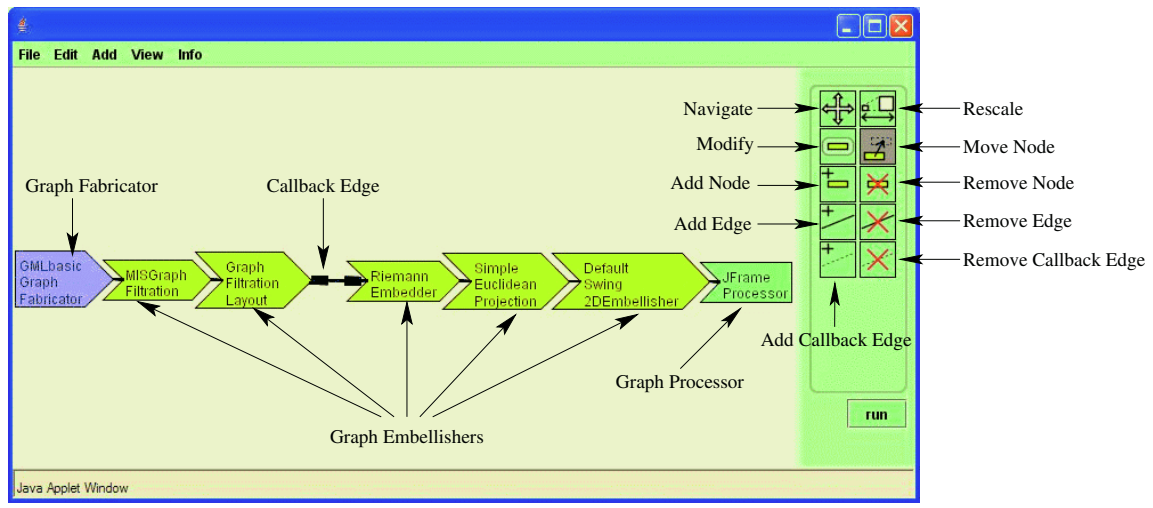

Fig. 3. A screenshot of the graphael CF-graph.

graph editor that can be accessed from within graphael. The editor is simple, but useful for users that do not have data to generate a graph from. The graph editor is especially helpful in the testing of new components, since simple cases can be modeled easily in the editor. With a point/click/drag user interface, vertices and edges can be added, deleted, or moved.

\section{Control Flow Graph}

Here we describe another novel features of graphael, the Control Flow (CF) graph $^{1}$. The CF-graph allows the user to put together different combinations of layout algorithms, projections and visualizations, while offering a visual representation of the derived graph's production process; see Fig. 3. CF-graphs contain CF-nodes (such as layout algorithms) that act to generate and refine a derived graph, and CF-edges that represent the channels of input and output, internally passed between the CF-nodes.

CF-graphs are created by adding new CF-nodes and connecting them with edges. Once a complete chain of appropriate CF-nodes has been completed, the "run" button activates all the graph fabricators (described below). Modifying how an existing $\mathrm{CF}$-graph produces derived graphs can be done by manipulating the composition of the current $\mathrm{CF}$-graph or by changing the internal properties of the production units of CF-nodes.

\subsection{CF-Nodes}

There are three different types of production units: fabricators, embellishers, and processors. Each of them is briefly described below.

1. Graph Fabricators are graph production units that take no input from other CF-graph entities. They act as the starting point for the generation of derived

\footnotetext{
${ }^{1}$ Note: For clarity we shall call a Control Flow graph a CF-graph and a graph produced
} by a CF-graph a "derived" or "production" graph. 
graphs since are not dependent on a derived graph as input. Many of the current graph fabricators available in graphael create the most raw form of a derived graph (i.e., vertex and edge declarations) by reading input files.

2. Graph Embellishers are methods that require a single, derived graph element as input from within the CF-graph and output a newly augmented, derived graph. In many cases, graph embellishers are used to add or modify properties of the derived graph they receive as input. For example, an embellisher could take a weighted graph as input and produce a weighted graph in which the color property of the heaviest vertices makes them stand out.

3. Graph Processors are methods in the final stage in any CF-graph, since they do not pass derived graphs to other production units. These methods typically output the final derived graph in the form of a picture, or a file.

\subsection{Callback Edges}

In a CF-graph with normal edges, once a production unit is finished with its input, the graph is passed to the next unit until it reaches the end of the CFgraph. However, there are cases when this is not desirable. If we wish, for example, to show a graph layout in a series of iterations (as opposed to just the final product), we would require the use of graphael's callback edges. These edges allow the source CF-node to suspend its execution and pass the graph to the remainder of the $\mathrm{CF}$-graph, starting at the target of the callback edge. Once this finishes, execution resumes where the source CF-node left off. Using callbacks, we implemented features such as animation. Specifically, a layout that needs to iterate over the graph multiple times can suspend itself to let the resulting graph from each iteration reach the processor and be displayed on the screen. After the processor finishes, the layout runs the next iteration. Callback edges can be identified as thick, dotted lines.

\subsection{CF-Node Property Management}

Whereas the panel in Fig. 3 offers different ways to manipulate the CF-graph, the individual CF-nodes can be manipulated as well. Recall that one of the two ways to modify how a derived graph is produced is to change CF-node properties. While we do allow for customized property managers, we have implemented an automatic GUI generator to minimize the amount of work required to make additions to the graphael library. The GUI generator is implemented using Java's reflection capabilities. This allows graphael to dynamically examine methods and data members of Java classes that have been added to its library. The system detects which properties can be modified by looking for a pair of getter and setter methods that meet certain conditions.

\section{Conclusion}

The graphael system has been implemented in Java, and is can be used to draw static graphs and evolving graphs online or offline. It can also be used as 
visualization platform for tools that generate graphs as output. For example, a database that produces graphs, such as the one described in [5] can be coupled with graphael to provide visual interaction with the graphs. Our system currently supports the (graph markup language) file format. Simple modifications to the standard format accommodate vertex-weights and edge-weights, as well as timeslice information.

\section{References}

1. D. Auber. Tulip - a huge graph visualization framework. In M. Jünger and P. Mutzel, editors, Graph Drawing Software, pages 105-126. Springer-Verlag, 2003.

2. V. Batagelj and A. Mrvar. Pajek - analysis and visualization of large networks. In M. Jünger and P. Mutzel, editors, Graph Drawing Software, pages 77-103. SpringerVerlag, 2003.

3. S. S. Bridgeman, A. Garg, and R. Tamassia. A graph drawing and translation service on the www. International Journal on Computational Geometry and Application, 9(4-5):419-446, 1999.

4. T. Dwyer and P. Eckersley. Wilmascope - a 3d graph visualization system. In M. Jünger and P. Mutzel, editors, Graph Drawing Software, pages 55-75. SpringerVerlag, 2003.

5. C. Erten, P. J. Harding, S. G. Kobourov, K. Wampler, and G. Yee. GraphAEL: Graph animations with evolving layouts. In 11th Symposium on Graph Drawing, pages 98-110, 2003.

6. C. Erten, P. J. Harding, S. G. Kobourov, K. Wampler, and G. Yee. Exploring the computing literature using temporal graph visualization. In Visualization and Data Analysis, pages 45-56, 2004.

7. T. M. J. Fruchterman and E. M. Reingold. Graph drawing by force-directed placement. Softw. Pract. Exper., 21(11):1129-1164, 1991.

8. P. Gajer and S. G. Kobourov. GRIP: Graph drawing with intelligent placement. Journal of Graph Algorithms and Applications, 6(3):203-224, 2002.

9. M. Jünger and P. Mutzel, editors. Graph Drawing Software. Springer-Verlag, 2003.

10. T. Kamada and S. Kawai. An algorithm for drawing general undirected graphs. Inf. Process. Lett., 31(1):7-15, 1989.

11. S. G. Kobourov and K. Wampler. Non-Euclidean spring embedders. In 10th Annual IEEE Symposium on Information Visualization (InfoVis). To appear in 2004.

12. J. Lamping, R. Rao, and P. Pirolli. A focus+context technique based on hyperbolic geometry for visualizing large hierarchies. In Proceedings of the SIGCHI conference on Human factors in computing systems, pages 401-408, 1995.

13. S. B. Seidman. Network structure and minimum degree. Social Networks, 5:269287, 1983.

14. R. Wiese, M. Eiglsperger, and M. Kauffmann. yfiles - visualization and automatic layout of graphs. In M. Jünger and P. Mutzel, editors, Graph Drawing Software, pages 173-192. Springer-Verlag, 2003. 